\title{
Medical Staff's Opinions Regarding Factors which Influence the Case Management of Children with Insulin-dependent Diabetes Mellitus
}

\author{
Lica Melania Maria ${ }^{1,4^{*}}$, Jakab Zoltan², Crainic Maria ${ }^{3,5}$, Mihai Adriana ${ }^{4,5}$ \\ Clinical Psychology, Paediatric Department, Diabetic Children and Youth Association Mures, Romania \\ 2 Mental Health Centre Tg Mures, Romania \\ 3 University of Medicine and Pharmacy luliu Hatieganu, Cluj Napoca, Romania \\ 4 Psychiatric Department, University of Medicine and Pharmacy Tirgu Mures, Romania \\ 5 Institute of Psychotherapy and Personal Development, Tirgu Mures, Romania
}

Insulin-dependent diabetes mellitus (IDDM) is a chronic disease with high incidence, especially in children, and for a better outcome the individual case management is required. The patient and his family can experience different levels of anxiety, with negative effects on disease evolution and prognosis. Objectives: identify relevant factors which influence the case management of children with IDDM. The implementation of the results of this study will help to elaborate an efficient method of intervention for improving the treatment adherence and obtaining a better outcome of IDDM in children. Methods: A semi-structured interview was done individually to 10 experts in diabetes for collecting their opinion concerning relevant factors that may influence treatment adherence and what are the characteristics of an efficient method of intervention. Results: Majority of experts considered that the main factor which influences the outcome is doctor-patient relationship. The need of child, to feel that it is understood and protected, was considered that can directly motivate a better self-management and a good outcome. The increase of importance of individual resources of children with IDDM and accent on the quality of life of those children could be considered a starting point for creating a psychological clinic intervention in this field. The results were compared with scientific literature data. Conclusions: understanding of the causes which have interfered negatively with therapeutic plan is a starting point for develop a clinical psychological protocol for children with IDDM.

Keywords: insulin-dependent diabetes mellitus in children, adherence to treatment, psychological intervention

Received: 10 March 2016 / Accepted: 01 August 2016

\section{Introduction}

Insulin dependent diabetes mellitus (IDDM) is a chronic disease that affects more children in Romania and worldwide, considered to be the most common chronic disease of children. In 2014 were 3014 children with IDDM in Romania (61\% urban) [1]. According to the World Health Organisation WHO - there are 240 million people with diabetes in the world, most cases found in Europe and North America; at the opposite pole are Japan, Central and South America [2]. For insulin-dependent patient the diabetes means: continuous monitoring of blood glucose, injecting insulin, determination of carbohydrates, regular meals, supervised sports activity and other regular specialist controls in Neurology, Nephrology, Ophthalmology, Endocrinology, Podiatry; in order to prevent micro- and macro-angiopathy complications. On the other side, every-day life involves a good self-control, organization, responsibility and self-motivation. This is like a complete job, where an individual cannot be absent or late, because of the acute risk for his own life and specific disease complications.

So, these continuous perceived responsibilities create a source of stress, which is able to disturb the emotional balance of child and his parents.

* Correspondence to: Melania Cozma

E-mail: melanialica@yahoo.com
From our experience with diabetes patients, medical prescription such as blood sugar measurement, insulin injection, diet and physical exercises doesn't mean only that the patient will have appropriate expected results, but also involves an important change of life. This involve the reorganization of habits, a change of thoughts and emotional adjustment both to the child and his family, in order to accept the existence of his disease and its impact on own life and adapting to the conditions imposed by it.

In this research, we studied the effect of various factors involved into patient-disease relationship and the conditions for a better adherence to the therapy. In the literature it is a clear distinction between compliant and adherence [3]. In paternalistic style of doctor-patient relationship we expected from the patient to be obedient, to listen, believe and put in practice doctor's orders, nowadays the new style consist in empowering the patient and family in process of care [4]. In the past was a very tide connection between the patient and the physician, which guide the patient toward wellbeing. All responsibility was on doctor's hands and the patient's health state, depend on that fact. The changes appeared when the physician started to be interested in patient personal satisfaction and the role of this independent variable in defining quality of life of individuals with chronic disease.

Treatment adherence is a long-term goal, important for defining thequality oflife, butalso a continuing challenge [5]. 
There is well known the impact of a low-adherence on treatment plan, on diabetes control, with very high or very low blood sugar values, coma, diabetic ketoacidosis, unbalanced specific diabetic complications as neuropathy, nephropathy, retinopathy, etc [6]. The goal of this study was to identify relevant factors, which influence the case management of children with IDDM. The implementation of the results of this study will help to elaborate an efficient method of intervention for improving the treatment adherence and obtaining a better outcome of IDDM in children.

\section{Methods}

The study design was a qualitative descriptive one, using the method of a semi-structured interview on a homogenous group of experts.

Specialists on IDDM in children and teenagers from our area were contacted and informed about our interest on a research about the factors, which influence the case management of IDDM. They were asked to participate at one-hour interview on this topic [7].

Ten experts in Diabetes accepted to participate (71.42\%). The doctor involved are all specialist in Diabetes, with more than 5 years experience in this field, working in public or private hospital with children with insulin dependent diabetes mellitus, and expressing their accord for this interview. There was not any conflict of interests or any material benefits from participating of this study, no name or personal data regarding patient or doctors were mentioned in this study.

A semi-structured interview was applied in order to collect expert's opinion concerning relevant factors that may influence the case management, treatment adherence and the characteristics of an efficient method of intervention.

The interview was done by a specialist in psychology, doctoral student, member in the project. Because all inter-

Table I.

\begin{tabular}{lc}
\hline $\begin{array}{l}\text { Main factors, ordered by priorities by specialists, } \\
\text { considered important in management of the case in } \\
\text { children with diabetes type 1 }\end{array}$ & $\begin{array}{c}\text { The rate of } \\
\text { response }\end{array}$ \\
\hline 1. Doctor-patient relationship & $100 \%$ \\
2. Quality of communication with children and their & $100 \%$ \\
parents & $100 \%$ \\
3. Self-monitoring of daily blood-sugar values & $100 \%$ \\
4. Children's and parent's knowledge about diabetes & $100 \%$ \\
5. Doctor's knowledge about diabetes & $100 \%$ \\
6. Number of tests available for measure of blood-sugar & $90 \%$ \\
7. Time assigned for consultation & $90 \%$ \\
8. Need for psychological intervention & $80 \%$ \\
9. Children's family problems & $70 \%$ \\
10. Children's motivation & $30 \%$ \\
11. Burn -out syndrome & $20 \%$ \\
12. Doctor's psychological knowledge & $10 \%$ \\
13. A psychologist involvement in case management & $0 \%$ \\
14. Doctor's participation on psychological workshops & $0 \%$ \\
15. Group therapy & $0 \%$ \\
16. Other the methods of intervention ( suggestions) &
\end{tabular}

views were done by the same person the bias related with interview procedure, interviewing skills or interviewer personality traits could be excluded. Interviews were done between $1^{\text {st }}$ October 2015 and 30 $0^{\text {th }}$ November 2015. Each interview lasted on average 50 minutes. The interview was done in calm and isolated room. If the expert refused to be audio-recorded the written notes were taken during the interview. The audiotapes were deleted in the same day after the collection of information on written notes. We didn't record any confidential data for avoiding the identification of the expert.

The items evaluated during the interview were: professional experience with children with IDDM, frequency of specialized visits, situations that require hospitalization of children with IDDM in the Pediatric Clinic, collaboration with physicians in other specialities, their point of view on the factors able to produce a high adhesion, and causes identified as responsible for the poor adherence to the therapy, methods and tools used by them to monitories the patients, indicators of adherence and non-adherence, recorded in patient charts, their own strategies used in their practice to reduce the phenomenon of non-adherence, type of interaction with patients and their parents (directindividual, group meetings), problems that they face with in their professional activity, attend professional training programs, participation in specialized conferences, implementation of innovative strategies in clinical practice, availability to be contacted by children with IDDM and their families between consultations (every 3 months), referral occurrence of psychological disorders and initiative to seek the counseling services in those cases, etc.

\section{Results and discussions}

Looking at the compliant behaviour of children with IDDM, one of the involved specialists in diabetes claimed that "there are differences from child to child", and "is very hard to outline a hierarchy of factors that influence treatment adherence".

In specialist opinion the main factor was considered doctor - patient relationship (Table I). The need of the child to be understood and protected was considered that directly motivate a better self-management and a good evolution of this chronic disease. Even when the child comes from a disadvantaged social background, from a broken family, or one with limited educational and financial resources, the doctors had note an important behavioural changes after some days of hospital recovery, if they succeed in a good relationship with the children and their families. Most of the time the contact with the patient was during the individual consultation, face to face; other actors have been involved in the doctor - patient relation-ship only during hospitalization, such as nurses, diet specialists, other staff. All specialists have recognized as a priority the communication with children and their parents. They believe that this is an important factor in adherence to treatment; "the importance of establishing a trusting relationship with parents" 
is reflected in various new situations or problems appeared, in an early information of the doctor, for a better decision (eg." the patients and their parents had to know the symptoms, the insulin type, the doses, the values of laboratory tests" etc ). The doctors encourage self-monitoring of blood-sugar values, food and insulin ratio, and note them in a personal notice book, in addition to the clinical patient observation sheet, and "always try to explain the details to the patient or/and family, before to ask them to do some change in the therapeutically plan". In this way there are no dubs about the importance of the compliant behaviour, increasing motivation to participate to the success of the therapy. This type of combined monitoring helps both the specialist and the patient to know the real state of the disease and the responsibility to an appropriate treatment. They associate this item with doctor-patient relationship, because, according to the doctors experience, "the patients or/and their parents with communication difficulties, have problem to follow the self-monitoring and daily recording request, or writing false-ideal values". They also have founded" cases requiring psychological counselling", especially when these problems interferes with diseasemanagement, and recognize a "better adherence after intervention". Regarding this, a "real problem is the absence of a psychologist hired to evaluate / council psychologically the children with IDDM and usually only children whose parents can afford a private consultation can benefit from immediate intervention focused on problem". Also, 8 specialists have noticed a relation between low adherence to the diabetes therapy and problems related to their family.

All doctors gave children and parents informative materials, inform them about how to calculate carbohydrates, insulin ratio related glucose value, but there are significant differences between case management. They feel, "frustrated" that some patient or their parents are giving more credibility to "internet forums" or "good friends advises" like source of information, many time incomplete or wrong, increasing the risk of complication, or even death. This fact is seen as a decreasing of therapeutically confidence, a starting point for errors and collateral damages. About the professional training, all doctors regularly read and write articles, participate to diabetes courses and congresses, only based on medical approach, without psychological information or assertive communication skills. Two of them read also psychological article but regarding general information, not especially about diabetes subjects.

Among the issues raised by the medical physicians are the insufficient blood sugar tests, with negative consequences on self-monitoring of diabetes, and sometime used as an excuse by the patient or/and parents for missing values in their diabetes diary. Another issue is the short time assigned for a consultation ( $15 \mathrm{~min})$ divided for clinical examination, measurement of blood sugar, medical counselling, nutrition, etc. There are exceptions, when the children are hospitalized and the doctors have more time to analyse general health and special problems in diabetes management. Being admitted in a hospital is an important stress for a child and also in this case the patient is observed in artificial life condition, which is different comparing with "real-life". Another problem that 3 of diabetes doctors have noticed was "the feeling of helplessness" that they were feeling in most unbalanced cases. The worrying laboratory results (high level of glycosylated haemoglobin, severe oscillations of blood sugar values, early appearance of diabetes specific complications) do not reflect their work and involvement. These unsuccessful situations generated feelings of dissatisfaction and a slight decline of "motivation to be emotionally involved in relationships with their patients". On the other hand glycemic control and a good general health condition of the patients, a high adherence to the therapeutic indications, it seems to be proportion to their confidence on specialist skills. Even one of the doctors said that "therapeutically success does not depend only by the physician, this responsibility is divided", they perceive the full responsibility for every case. These results reflect the need of psychological support in order to avoid burnout, such as group counselling for specialists, Balint group, individual psychotherapy and other activities able to reduce negative effects of professional stress and to increase motivation to work with children with IDDM. The accent on individual resources, both on children's with IDDM and doctors, and increasing the importance of quality of life in diabetes management, could be considered a starting point for creating a psychological clinic intervention in this field. The results will be compared with literature data in a future study with a retrospective research on treatment adherence of children with IDDM.

From literature the factors that may influence treatment adherence are divided in socioeconomic factors, patient's age, cultural and educational factors, psychological factors, health system and history of the disease [8]. Regarding this, Osterberg in 2005 described a triangular pattern of dynamic factors, consisting in patient-health carephysician relationship, about how difficult is to improve a higher adherence, and about the cost of low adherence ("worsening of the disease, and increased health care cost, even death") [9]. The patient factor refers to the severity of the disease, years in diabetes, complications, signs, his conception about the disease, how he cope with the situation, "the patient's diary", if he trust in doctor, etc. About the treatment, his attention is focused on how long is the necessity to do the therapy, potentials collateral damages, details about the administration, costs, etc. In his opinion, an important actor of the long term therapy is the physician; talking about the doctor's diary" he had outlined "the goals this wants to achieve" by his intervention and the "ability to communicate them to the patient". Mainous was highlighting also the importance of therapeutic alliance, based on trust and safety [10]. He talk about "adherence of white smock", fact that was recognized in medical practice, when a lot of patients has compliant therapeutic behaviour " 5 days before and 5 days after the consult". 
The ability of an experienced doctor is "to convince the patient to extend this period".

The first authors who spoke about the doctor patient relationship in chronic diseases were Szasz and Hollender in 1956, emphasizing the effectiveness of this through "mutual participation.... which helps the patient to help itself" [11]. Even though in certain stages of the disease (eg. in diagnosis moment, in critical situation of the disease), the patient needs to be redirected, his active involvement in the management of disease is a sure evidence of development of individual ability to take action consciously and responsible for own health, on long-term. "Advisory Board" (groups of working- specialists from Italy, Sweden, Denmark) have tried to highlighting ,variables" that determine a degree of adherence, and the practical applications of the results [12]. Their methods were: interviewing the patients, diaries, counting unused drugs from the prescription and clinic evaluation. The conclusion was: "To know how much the patient has joined, a global approach is required" and a combination of all methods of analysis, and like other experts proposed "multidisciplinary pilot studies".

DCCT (Diabetes Control and Complication Trial) and Eurodiab conducted a study focused on disease management, including education, the psychological inference and self-control $[13,14]$. In Western countries, 50\% of patient with diabetes mellitus were characterized with poor adherence), explaining in this way the cause of ineffective treatment with high impact on individual and social level. Regarding the chronic medical situation, Loonen (2002) use the "forced cohabitation" term, that "interferes with the perception of their own inefficiency in relation to disease (stress and anxiety generating conflict)" [7]. This condition has to be a good moment to reconsider the patient-child with diabetes mellitus, and to put together, on the same level the diabetes medical suggestions, with psychological intervention focused on increasing self-esteem and perceived self-efficacy.

Figure represented by medical staff has in general a negative connotation for children with insulin depended diabetes mellitus (IDDM), related to several injections, restrictive diet, pricking many time in a day for blood glucose measurement; some children get to gradually release from this authority, trying to assert autonomy and freedom opposing medical prescription, or falsifying data from blood glucose diary specifications. Some parents have an attitude that defies the medical methods, or refuse to administrate insulin to child, because they heard about different therapies. On short and long terms all these behaviours have negative health effects on children with diabetes. The most common identified factors that influence the therapeutical adherence of children with diabetes is the human factor, doctor-patient relationship, the source of quality information and emotional support, in order to increase motivation for a better self-management. The doctor could investigate the goals of the patient, the motivation, to find out what kind of feelings he has and what is expecting from the therapy, from medical staff and how he cope with diabetes in family, school and social environment.

There are a lot of objective and subjective problems that interferes with every-day life of the children with diabetes mellitus, with consequences in medical, psychological and social field, and it is very important to discover which is a potential enemy to their adherence to the therapy and their personal coping with the situation. Another detail regarding this is to find out the children's individual and families resources, focus on quality of life.

Adherence therapy decreases with age in diabetes, especially in adolescence, so this must be included in the research about factors involved in case management and discover how they are acting, a possible circularity; It is considered that psychological comfort and confidence is a state against which the child can learn, express needs, fears, and organise the own life, like other children without diabetes, and this is a real resource for increasing the selfrecognition.

Having a positive constructive attitude, a dialogue from the same level, building an open, secure, trustworthy relationship with the child and family, leads to a better adherence to diabetes on long term. "The confidence is an ethical dimension of medical care", said Sandu A. in 2013 [15].

"Children learn what they live... If children live with encouragement, they learn confidence... If children live with recognition, they learn it is good to have a goal" said Low Doroty Nolte [16]. The children with diabetes are experiencing different challenges, in different environment, with different people, at home, at school, in doctor office, with friends, and a good attitude is to teach them how to use their personal resources to cope better with their situation. In diabetes, there is a circular relation between somatic-psychological and social aspects, so is necessary to include in clinical evaluation the bio-psycho-socials factors which influence treatment adherence. Taking care about children means to use an appropriate language to make them understand, and to treat them with respect, talking to second person, without judgment and damnation. The base of a good management seems to be the open way to communicate, so it would be important that the doctors could practice personal development sessions, to be able to recognize and overcome their vulnerabilities, fears or frustrations in relation with children with diabetes and their families, condition that could produce maladaptive reactions, source of perceive stress and interpersonal-conflict, a circular relation between feelings and behaviour. Understanding of the causes, which have interfered negatively with therapeutic plan, is a starting point for develops a clinical psychological protocol for children with IDDM.

The main objective of this study was to outline an overview which of specialist doctors in diabetes have formed from their experiences with children with IDDM, regarding the factors, which, in their opinion, have an important contribution in, determinate the adherence to the diabetes 
therapy. Comparing the results from semi-structured interview with the literature, it was a first step towards understanding negative behaviours, as poor compliance to treatment, with negative effects for both patients and for the external costs that is generating for the health system.

The limit of this study was the number of the specialist involved, not including on interview process nurses, an important actors especially during hospital recovery. It will be also important to study, in a future research, the perception of the children and their family regarding the disease and the own difficulties to cope with it.

\section{Conclusions}

The study's results highlights the importance of relations between child with diabetes and the medical authority, seen as a knowledge base and emotional support, as a joint project partners, to better management of the disease, on long term. As specialists in diabetes have revealed, it is not a single way to look at a chronic disease, every detail could make the difference in patient approach. It is important to create the opportunity to increase the standard of professional attitude in relation with children with diabetes and their families. Creating a safe relationship, based on trust, personal diversity acceptance, patient dignity respect and an open communication, considering as important every detail related to personal and/or family's issues in disease management, is an optimistic premise for a long term therapies success and a high quality of life. A professional attitude could be complete only with an appropriate space and right time assigned to a specialist consultation, involving in treatment plan other medical staff as other specialist, nurses, nutritionist, including always the child's family. Sharing responsibility could be an opportunity to get viable results and a good way to reduce doctor's emotional exhaustion.

The patient does not belong to the medical system, but assisted by it, and when he steps outside hospital has to be certain on finding professional support when needed. In this condition, the responsibility is divided between medical system and the child's family, who became partners for all life, from the first moment, the diagnosis. All this conclusions suggests the need to build a practical protocol applied in this partnership that should be focused on history of the case including physical, psychological and social aspects of children with diabetes, suggestion of intervention focused on identified barriers in the disease management and structured educational therapy, other specialist interventions, results and suggestions. The strengths of this protocol is to facilitate a better relationship between medical system and patient, a good management of risk situation and an early intervention on issues, with consequence in reducing the social and medical cost and a better quality of life.

\section{Conflicts of interest}

The authors report no conflicts of interest.

\section{References}

1. Romanian Society of Diabetes, Nutrition and Metabolic Diseases. PREDATORR Study, 2014. www.societatediabet.ro/comunicat\%20 rezultate\%20PREDATORR\%20\%2021\%20mai\%202014.php.

2. World Health Organization. Global Health Estimates: Deaths by Cause, Age, Sex and Country, 2000-2012. Geneva, WHO, 2014. http://www. who.int/healthinfo/global_burden_disease/estimates/en/index1.html

3. Majani G. Compliance, adesione, aderenza. I punti critici della relazione terapeutica. Ed. McGraw - Hill, Publishing Group Italia, Milano, 2001, pg. 20-190.

4. Matthews E, Can paternalism be modernised? J Med Ethics. 1986;12(3):133-135.

5. Rapoff MA. Rapoff M A. Adherence to pediatric medical regimens. 2nd edn. New York: Springer; 2010.

6. Ellis DA, Naar-King S, Frey M, Rowland MD, Greger N. Case study: Feasibility of multisystemic therapy as a treatment for urban adolescents with poorly controlled type 1 diabetes. J Pediatr Psychol. 2003;28(4):287293.

7. Van der Zaag-Loonen HJ, Grootenhuis MA, Last BF, Derkx HHF. Coping Strategies and Quality of Life of Adolescents with Inflammatory Bowel Disease. Qual Life Res. 2004;13(5):1011-1019.

8. Warren L, Hixenbaugh P. Adherence and Diabetes. Adherence to Treatment in Medical Conditions (Edited by: Meyers LB, Midence K). Amsterdam. Harwood. 1998 pg 423-453.

9. Osterberg L, Blaschke T. Adherence to medication. $N$ Engl J Med. 2005;353(5):487-497.

10. Mainous AG, Goodwin MA, Stange KC. Patient-physician shared experiences and value patients place on continuity of care. Ann Fam Med. 2004;2:452-454.

11. Szasz TS, Hollender MH. A contribution to the philosophy of medicine; the basic models of the doctor-patient relationship. AMA Arch Intern Med. 1956;97:585-592.

12. Peyrot M1, Rubin RR, Lauritzen T, et al. Resistance to insulin therapy among patients and providers: results of the cross-national Diabetes Attitudes, Wishes, and Needs study (DAWN). Diabetes Care. 2005;28:2673-2679.

13. DCCT Research Group. Implementation of treatment protocols in the Diabetes Control and Complications Trial. Diabetes Care. 1995;18(3):361376.

14. Green A, Gale E, Patterson CC. Incidence of childhood-onset insulindependent diabetes mellitus: the EURODIAB ACE study. Lancet. 1992;339(8798):905-909.

15. Sandu A, Cojocaru D, Gavrilovici C, Oprea L. Trust: An ethical dimension of healthcare in chronic disorders. Romanian Bioethical Journal. 2013;11(1):190-204.

16. Low Doroty Nolte. Children Learn What They Live, www. EmpowermentBooks.com/Vooks for Parents, 4/17/97

17. Davis DA, Taylor- Vaisey A. Translating guidelines into practice: a systematic review of theoretic concept, practical experience and research evidence in the adoption of clinical practice guidelines. Can Med Assoc Journal. 1997;157(4):408-416.

18. Cochrane LJ, Olson CA, Murray S, et al. Gaps between knowing and doing: Understanding and assessing the barriers to optimal health care. J Contin Educ Health Prof. 2007;27(2):94-102.

19. Perkins MB, Jensen PS, Jaccard J, Gollwitzer P, Oettingen G. Applying Theory- Driven Approaches to Understanding and Modifying Clinicians' Behavior: What Do We Know Psychiatr Serv. 2007;58(3):342-348.

20. Wagner EH, Austin BT, Von Korff M. Organizing care for patients with chronic illness. Milbank Q. 1996;74(4):511-544.

21. Stott $\mathrm{NCH}$, Rollnick S, Rees MR, Pill RM. Innovation in clinical methods: diabetes care and negotiating skills. Fam Pract. 1995;12(4):413-418.

22. Duggan PS, Geller G, Cooper LA, Beach MC. The moral nature of patient centeredness: is it "just the right thing to do"? Patient Educ Couns. 2006;62(2):271-276.

23. Quill TE, Brody H. Physician Recommendations and Patient Autonomy: Finding a Balance between Physician Power and Patient Choice. Ann Intern Med. 1996;125:763-769. 